\title{
Vacuum Energy Density in the Quantum Yang-Mills Theory
}

\author{
G.G. Barnaföldi ${ }^{1,2}$ and V. Gogokhia ${ }^{1}$ \\ ${ }^{1}$ HAS, CRIP, RMKI, Department of Theoretical Physics, Budapest 114, P.O.B. 49, \\ H-1525, Hungary \\ ${ }^{2}$ Center for Nuclear Research, Department of Physics, Kent State University, Kent, \\ Ohio 44242, USA \\ E-mail: bgergely@rmki.kfki.hu, gogohia@rmki.kfki.hu
}

\begin{abstract}
Using the effective potential approach for composite operators, we have formulated a general method of calculation of the truly non-perturbative Yang-Mills vacuum energy density (this is, by definition, the bag constant apart from the sign). It is the main dynamical characteristic of the QCD ground state. Our method allows one to make it free of the perturbative contributions ('contaminations'), by construction. We also perform an actual numerical calculation of the bag constant for the confining effective charge. Its choice uniquely defines the bag constant, which becomes free of all the types of the perturbative contributions now, as well as possessing many other desirable properties as colorless, gauge independence, etc. Using further the trace anomaly relation, we develop a general formalism which makes it possible to relate the bag constant to the gluon condensate defined at the same $\beta$ function (or, equivalently, effective charge) which has been chosen for the calculation of the bag constant itself. Our numerical result for it shows a good agreement with other phenomenological estimates of the gluon condensate. We have argued that the calculated bag constant may contribute to the dark energy density. Its contribution is by 10 orders of magnitude better than the estimate from the Higgs field's contribution. We also propose to consider the bag energy as a possible amount of energy which can be released from the QCD ground state by a single cycle. The QCD ground state is shown to be an infinite and hence a permanent reservoir of energy.
\end{abstract}

PACS numbers: 11.15.Tk, 12.38.Lg, 12.38.Aw 


\section{Introduction}

In order to calculate physical observables from first principles in Quantum Chromodynamics (QCD) [1] it is not enough to know its Lagrangian. It is also necessary and important to know the true structure of its ground state. It is the response of the QCD vacuum which substantially modifies all the QCD Green's functions from their free counterparts. These full ("dressed") Green's functions are needed for the above-mentioned calculations. The vacuum of QCD is a very complicated confining medium and its dynamical and topological complexity means that its structure can be organized at various levels: classical and quantum [1, 2, 3, 4, 5, 6] (and references therein). It is mainly non-perturbative (NP) by origin, character and magnitude, since the corresponding coupling constant is large. However, the virtual gluon field configurations and excitations of the perturbative (PT) origin, character and magnitude, due to asymptotic freedom (AF) [1], are also present there.

One of the main dynamical characteristics of the QCD ground state is the bag constant. Its name comes from the famous bag models for hadrons [7, 8], but its present understanding (and thus modern definition) not connecting to hadron properties. It is defined as the difference between the PT and the NP vacuum energy densities (VEDs) [9, 10, 11, 12]. So, we can symbolically put $B=V E D^{P T}-V E D$, where $V E D$ is the NP but 'contaminated' by the PT contributions (i.e., this is a full $V E D$ like the full gluon propagator, see below). At the same time, we can continue as follows: $B=V E D^{P T}-V E D=V E D^{P T}-\left[V E D-V E D^{P T}+V E D^{P T}\right]=V E D^{P T}-\left[V E D^{T N P}+\right.$ $\left.V E D^{P T}\right]=-V E D^{T N P}>0$, since the $\mathrm{VED}$ is always negative. The bag constant is nothing but the truly NP (TNP) VED, apart from the sign, by definition, and thus is free of the PT contributions ('contaminations'). The symbolic subtraction presented here includes the subtraction at the fundamental gluon level, and two others at the hadronic level, i.e., when the gluon degrees of freedom should be integrated out (see section 3 below). In order to consider it also as a physical characteristic of the QCD ground state, the bag constant correctly calculated should satisfy some other necessary requirements such as colorlessness, finiteness, gauge-independence, no imaginary part (stable vacuum), etc.

The main purpose of this paper is to formulate a formalism how to calculate correctly the quantum part of the bag constant, using the effective potential approach for composite operators [13, 14, 15]. In particular, to show how the above-mentioned subtractions are to be analytically made. On account of the confining effective charge, the bag constant has been numerically evaluated, satisfying all the necessary requirements mentioned above. Using further the trace anomaly relation [16, 17, 18, 19], we also develop a general formalism which makes it possible to relate the bag constant to another important NP characteristic of the QCD ground state - the gluon condensate [11. Here, we do not use the weak coupling solution for the corresponding $\beta$ function. Finally we present our numerical result for the bag constant, which is in a good agreement with other phenomenological estimates of the gluon condensate [11, 20]. 


\section{The VED}

The quantum part of the VED is determined by the effective potential approach for composite operators [13, 14, 15]. In the absence of external sources the effective potential is nothing but the VED. It is given in the form of the skeleton loop expansion containing all the types of the QCD full propagators and vertices, see Fig. 1, So each vacuum skeleton loop itself is a sum of an infinite number of the corresponding PT vacuum loops (i.e., containing the point-like vertices and free propagators, see Fig. 2, where one term in each lower order is shown, for simplicity). The number of the vacuum skeleton loops goes with the power of the Planck constant, $\hbar$.

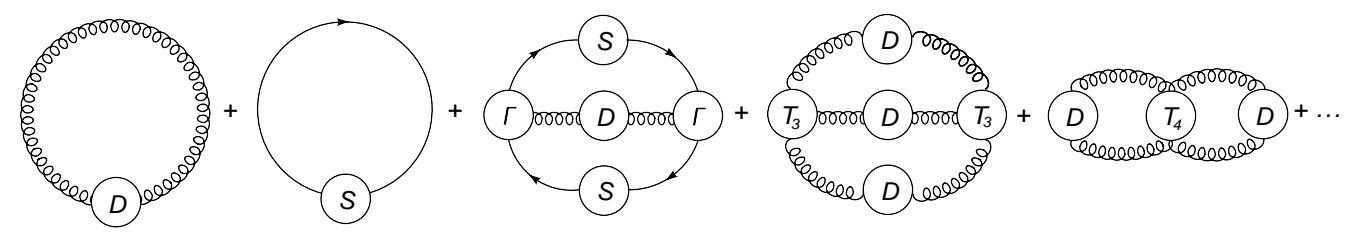

Figure 1. The skeleton loop expansion for the effective potential. The wavy lines describe the full gluon propagators $D$. The solid lines describe the full quark propagators $S$. $\Gamma$ is the full quark-gluon vertex, while $T_{3}$ and $T_{4}$ are the full three- and four-gluon vertices, respectively.

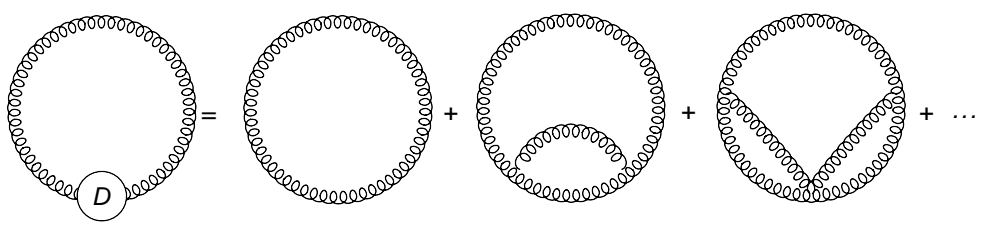

Figure 2. Infinite series for the gluon part of the VED (taking the first skeleton diagram in Fig. (1).

Here we are going to formulate a general method of numerical calculation of the quantum part of the TNP Yang-Mills (YM) VED in the covariant gauge QCD. The gluon part of the VED to leading order (the so-called log-loop level $\sim \hbar$, the first skeleton loop diagram in Fig. 1, and which PT expansion is shown explicitly in Fig. 2) is analytically given by the effective potential for composite operators as follows [13]:

$$
V(D)=\frac{i}{2} \int \frac{\mathrm{d}^{4} q}{(2 \pi)^{4}} \operatorname{Tr}\left\{\ln \left(D_{0}^{-1} D\right)-\left(D_{0}^{-1} D\right)+1\right\}
$$

where $D(q)$ is the full gluon propagator and $D_{0}(q)$ is its free counterpart (see below). The traces over space-time and color group indices are assumed. Evidently, the effective potential is normalized to $V\left(D_{0}\right)=0$, i.e., the free PT vacuum is normalized to zero, as usual. Next-to-leading and higher contributions (two and more vacuum skeleton loops) are suppressed at least by one order of magnitude in powers of $\hbar$. They generate very 
small numerical corrections to the log-loop terms, and thus are not important for the numerical calculation of the bag constant to leading order.

The two-point Green's function, describing the full gluon propagator, is

$$
D_{\mu \nu}(q)=-i\left\{T_{\mu \nu}(q) d\left(-q^{2} ; \xi\right)+\xi L_{\mu \nu}(q)\right\} \frac{1}{q^{2}},
$$

where $d\left(-q^{2} ; \xi\right)$ is the gluon invariant function (dimensionless), the so-called Lorentz structure (sometimes, we will call it as the full gluon form factor or, equivalently, the effective charge ("running"), see below), while $\xi$ is the gauge-fixing parameter and

$$
T_{\mu \nu}(q)=g_{\mu \nu}-\frac{q_{\mu} q_{\nu}}{q^{2}}=g_{\mu \nu}-L_{\mu \nu}(q)
$$

Its free PT counterpart $D_{0} \equiv D_{\mu \nu}^{0}(q)$ is obtained by putting the full gluon form factor $d\left(-q^{2} ; \xi\right)$ in Eq. (2) simply to one, i.e.,

$$
D_{\mu \nu}^{0}(q)=-i\left\{T_{\mu \nu}(q)+\xi L_{\mu \nu}(q)\right\} \frac{1}{q^{2}} .
$$

In order to evaluate the effective potential (1), on account of Eq. (2), we use the well-known expression

$$
\operatorname{Tr} \ln \left(D_{0}^{-1} D\right)=8 \times 4 \ln \operatorname{det}\left(D_{0}^{-1} D\right)=32 \ln \left[(3 / 4) d\left(-q^{2} ; \xi\right)+(1 / 4)\right],
$$

which becomes zero indeed when setting $d\left(-q^{2} ; \xi\right)=1$.

Going over to four-dimensional Euclidean space in Eq. (1), one obtains $\left(\epsilon_{g}=V(D)\right)$

$$
\epsilon_{g}=-16 \int \frac{\mathrm{d}^{4} q}{(2 \pi)^{4}}\left[\ln \left[1+3 d\left(q^{2} ; \xi\right)\right]-\frac{3}{4} d\left(q^{2} ; \xi\right)+a\right],
$$

where constant $a=(3 / 4)-2 \ln 2=-0.6363$ and the integration from zero to infinity over $q^{2}$ is assumed. The VED $\epsilon_{g}$ derived in Eq. (6) is already a colorless quantity, since it has been summed over color indices. Also it does not depend explicitly on the unphysical (longitudinal) part of the full gluon propagator due to the product $\left(D_{0}^{-1} D\right)$, which, in its turn, comes from the above-mentioned normalization to zero. Thus it is worth emphasizing that the transversal ("physical") degrees of freedom only of gauge bosons contribute to this equation. Note, in the effective potential approach to leading order there is no need for ghost degrees of freedom from the very beginning in order to cancel the longitudinal ("unphysical") component of the full gluon propagator. This role is played by the normalization condition (that is why the ghost skeleton loops are not shown in Fig. 1). Furthermore, overall numerical factor $1 / 2$ has been introduced into Eq. (1) in order to make the gluon degrees of freedom to be equal $32 / 2=16=8 \times 2$, where 8 color of gluons times 2 helicity (transversal) degrees of freedom, see Eqs. (5) and (므).

In the connection with the above-mentioned normalization condition a few remarks are in order. It does not work for the higher order vacuum loops. As explained in Ref. [13], for consistency with them in the PT QCD Green's functions, for example in the Hartree-Fock approximation, the Landau gauge should be used. In Ref. [21] the effective 
potential has been used to the two-loop order for the investigation of QCD chiralsymmetry breaking just in the Landau gauge and in the Hartree-Fock approximatiion. In the general case (i.e., beyond the PT and at any gauge), however, the cancelation of unphysical gluon modes should proceed with the help of ghosts as it is described in more detail in appendix A.

The derived expression (6) remains rather formal, since it suffers from the two serious problems: the coefficient of the transversal Lorentz structure $d\left(q^{2} ; \xi\right)$ may still depend explicitly on $\xi$. Furthermore, it is divergent at least as the fourth power of the ultraviolet (UV) cutoff, and therefore suffers from different types of the PT contributions.

\section{The TNP VED}

In order to define the VED free of the above-mentioned PT contributions ('contaminations'), let us make first the subtraction at the fundamental gluon level, namely

$$
d\left(q^{2} ; \xi\right)=d\left(q^{2} ; \xi\right)-d^{P T}\left(q^{2} ; \xi\right)+d^{P T}\left(q^{2} ; \xi\right)=d^{T N P}\left(q^{2}\right)+d^{P T}\left(q^{2} ; \xi\right)
$$

where $d^{P T}\left(q^{2} ; \xi\right)$ correctly describes the PT structure of the full effective charge $d\left(q^{2} ; \xi\right)$, including its behavior in the UV limit (AF, [1]), otherwise remaining arbitrary. On the other hand, $d^{T N P}\left(q^{2}\right)$ defined by the above-made subtraction, is assumed to reproduce correctly the TNP structure of the full effective charge, including its asymptotic in the deep infrared (IR) limit. This underlines the strong intrinsic influence of the IR properties of the theory on its TNP dynamics. Evidently, both terms are valid in the whole energy/momentum range, i.e, they are not asymptotics. Let us also emphasize the principle difference between $d\left(q^{2} ; \xi\right)$ and $d^{T N P}\left(q^{2}\right)$. The former is the NP quantity "contaminated" by the PT contributions, while the latter one being also NP, nevertheless, is free of them. Thus the formal separation between the TNP effective charge $d^{T N P}\left(q^{2}\right)$ and its PT counterpart $d^{P T}\left(q^{2} ; \xi\right)$ is achieved. For example, if the full effective charge explicitly depends on the scale responsible for the TNP dynamics in QCD, say $\Delta^{2}$ - the so-called mass gap (see section 5 below), then one can define the subtraction as follows: $d^{T N P}\left(q^{2} ; \Delta^{2}\right)=d\left(q^{2} ; \Delta^{2}\right)-d\left(q^{2} ; \Delta^{2}=0\right)=d\left(q^{2} ; \Delta^{2}\right)-d^{P T}\left(q^{2}\right)$, which is, obviously, equivalent to the decomposition (7). In this way the separation between the TNP effective charge and its PT counterpart becomes exact, but not unique. Let us emphasize that the dependence of the full effective charge $d\left(q^{2}, \Delta^{2}\right)$ on $\Delta^{2}$ can be only regular. Otherwise it is impossible to assign to it the above-mentioned physical meaning, since $\Delta^{2}$ can be only zero (the formal PT limit) or finite, i.e., it cannot be infinitely large. In principle, in some special models of the QCD vacuum, such as the Abelian Higgs model [22, 23], the NP scale is to be identified with the mass of the dual gauge boson.

There is also another serious reason for the subtraction in Eq. (7). The problem is that the above-mentioned UV asymptotic of the full effective charge may depend 
on the gauge-fixing parameter $\xi$ explicitly, namely to leading order $d\left(q^{2}, \xi\right) \sim_{q^{2} \rightarrow \infty}$ $\left(\ln \left(q^{2} / \Lambda_{Q C D}^{2}\right)\right)^{c_{0} / b_{0}}$, where the exponent $\left(c_{0} / b_{0}\right)<0$ explicitly depends on the gaugefixing parameter $\xi$ via the coefficient $c_{0}$ based on Ref. [1], and $\Lambda_{Q C D}^{2}$ is the QCD asymptotic scale parameter. In this connection let us note that AF being a physical phenomenon does not depend on the gauge choice (it takes place at any gauge), while the UV asymptotic of the corresponding Green's function may be still gauge-dependent. This is just explicitly shown above. Evidently, in the decomposition (7) just the PT part of the full effective charge will be responsible for this explicit dependence on the gauge choice. Subtracting it, we will be guaranteed that the remaining part will not depend explicitly on the gauge-fixing parameter (that is why the dependence on $\xi$ is not explicitly shown in $\left.d^{T N P}\left(q^{2}\right)\right)$. Let us note that if there is no exact criterion how to distinguish between the TNP and the PT parts in the full effective charge in Eq. (7) as described above, then it is possible from the full effective charge to subtract its UV asymptotic only. However, in this case the separation between the TNP and the PT parts will be neither exact nor unique. For how to make this separation exact and unique at the same time see section 5 .

Substituting the decomposition (77) into Eq. (66) and doing some simple rearrangements, one obtains

$$
\epsilon_{g}=-\frac{1}{\pi^{2}} \int \mathrm{d} q^{2} q^{2}\left[\ln \left[1+3 d^{T N P}\left(q^{2}\right)\right]-\frac{3}{4} d^{T N P}\left(q^{2}\right)\right]+\epsilon_{P T},
$$

where the trivial integration over the angular variables in Eq. (6) has been already done. Here $\epsilon_{P T}$ is

$$
\epsilon_{P T}=-\frac{1}{\pi^{2}} \int \mathrm{d} q^{2} q^{2}\left[\ln \left[1+\frac{3 d^{P T}\left(q^{2} ; \xi\right)}{1+3 d^{T N P}\left(q^{2}\right)}\right]-\frac{3}{4} d^{P T}\left(q^{2} ; \xi\right)+a\right] .
$$

It contains the contribution which is mainly determined by the PT part of the full effective charge, $d^{P T}\left(q^{2}, \xi\right)$. The constant $a$ should be also included, since it comes from the normalization of the free PT vacuum to zero.

However, this is not the whole story yet. The first term in Eq. (8), depending only on the TNP effective charge, nevertheless, assumes the integration over the PT region up to infinity. It also represents the type of the PT contribution, which should be subtracted as well. If we separate the NP region from the PT one, by introducing the so-called effective scale $q_{\text {eff }}^{2}$ explicitly, then we get

$$
\epsilon_{g}=-\frac{1}{\pi^{2}} \int_{0}^{q_{e f f}^{2}} \mathrm{~d} q^{2} q^{2}\left[\ln \left[1+3 d^{T N P}\left(q^{2}\right)\right]-\frac{3}{4} d^{T N P}\left(q^{2}\right)\right]+\epsilon_{P T}+\epsilon_{P T}^{\prime},
$$

where

$$
\epsilon_{P T}^{\prime}=-\frac{1}{\pi^{2}} \int_{q_{\text {eff }}^{2}}^{\infty} \mathrm{d} q^{2} q^{2}\left[\ln \left[1+3 d^{T N P}\left(q^{2}\right)\right]-\frac{3}{4} d^{T N P}\left(q^{2}\right)\right] .
$$

This integral represents the contribution to the VED which is determined by the TNP part of the full gluon propagator but integrated out over the PT region. Along with $\epsilon_{P T}$ 
given in Eq. (9) it also represents a type of the PT contribution into the gluon part of the VED (8), as mentioned above. This means that the two remaining terms in Eq. (10) should be subtracted by introducing the TNP YM VED $\epsilon_{Y M}$ as follows:

$$
\epsilon_{Y M}=\epsilon_{g}-\epsilon_{P T}-\epsilon_{P T}^{\prime},
$$

where the explicit expression for $\epsilon_{Y M}$ is given by the integral in Eq. (10).

Concluding, let us emphasize that both subtracted terms $\epsilon_{P T}$ and $\epsilon_{P T}^{\prime}$, strictly speaking, are not the purely PT, since along with the nontrivial PT effective charge

$d^{P T}\left(q^{2}\right)$ they contain the TNP effective charge $d^{T N P}\left(q^{2}\right)$ as well. So to call them the $\mathrm{PT}$ contributions is a convention. More precisely it is better to say that these terms are "contaminated" by the PT contributions. The above-mentioned necessary subtractions can be made in a more sophisticated way by introducing explicitly the ghost degrees of freedom (see appendix A).

\section{The bag constant}

The bag constant (the so-called bag pressure) is defined as the difference between the PT and the NP VEDs [9, 10, 11, 12. So in our notations for the YM fields, and as it follows from the definition by Eq. (12), it is nothing but the TNP YM VED apart from the sign, i.e.,

$$
\begin{aligned}
B_{Y M}=-\epsilon_{Y M} & =\epsilon_{P T}+\epsilon_{P T}^{\prime}-\epsilon_{g}= \\
& =\frac{1}{\pi^{2}} \int_{0}^{q_{e f f}^{2}} \mathrm{~d} q^{2} q^{2}\left[\ln \left[1+3 \alpha_{s}^{T N P}\left(q^{2}\right)\right]-\frac{3}{4} \alpha_{s}^{T N P}\left(q^{2}\right)\right],
\end{aligned}
$$

where from now on we introduce the notation

$$
d^{T N P}\left(q^{2}\right) \equiv \alpha_{s}^{T N P}\left(q^{2}\right),
$$

since $d^{T N P}\left(q^{2}\right)$ is the TNP effective charge $\alpha_{s}^{T N P}\left(q^{2}\right)$, as noted above. This is a general expression for any model effective charge in order to calculate the bag constant, or the TNP YM VED apart from the sign, from first principles. It is our definition of the TNP YM VED and thus of the bag constant. So it is defined as the special function of the TNP effective charge integrated out over the NP region (soft momentum region, $\left.0 \leq q^{2} \leq q_{e f f}^{2}\right)$. It is free of the PT contributions, by construction. In this connection, let us recall that $\epsilon_{g}$ is also NP, but 'contaminated' by the PT contributions, which just to be subtracted in order to get Eq. (13) from Eq. (10).

Comparing expressions (6) and (13), one comes to the following 'prescription' to get Eq. (13) directly from Eq. (6):

(i) Replacing $d\left(q^{2}\right) \rightarrow d^{T N P}\left(q^{2}\right)$ or equivalently, $\alpha_{s}\left(q^{2}\right) \rightarrow \alpha_{s}^{T N P}\left(q^{2}\right)$.

(ii) Omitting the constant $a$ which normalizes the free PT vacuum to zero.

(iii) Introducing the effective scale $q_{e f f}^{2}$ which separates the NP region from the PT one in the $q^{2}$-momentum space.

(iv) Omitting the minus sign for the bag constant. 
At this stage the bag constant defined by Eq. (13) is definitely colorless (colorsinglet) and free of the PT contributions ("contaminations"). Let us remind that it also depends on only transversal degrees of freedom of gauge bosons (gluons). All its other properties mentioned above (finiteness, positivity, no imaginary part, etc.) depend on the chosen effective charge, more precisely on its TNP counterpart. It is worth emphasizing once more that in defining correctly the bag constant, three types of the corresponding subtractions have been introduced. The first one - in Eq. (7) at the fundamental gluon level and the two others - in Eq. (12), when the gluon degrees of freedom were to be integrated out.

For actual numerical calculations of the bag constant via the expression (13) it is always convenient to factorize its scale dependence. For this purpose, let us introduce the dimensionless variable and the TNP effective charge as follows:

$$
\alpha_{s}^{T N P}\left(q^{2}\right)=\alpha_{s}^{T N P}(z), \quad \text { where } \quad z=\frac{q^{2}}{q_{e f f}^{2}} .
$$

From the general expression for the bag constant (13) in these terms one then gets

$$
B_{Y M}\left(q_{e f f}^{2}\right)=q_{e f f}^{4} \times \Omega_{Y M}
$$

where we introduce the dimensionless TNP YM effective potential $\Omega_{Y M}$, for convenience. Its explicit expression is

$$
\Omega_{Y M}=\frac{1}{\pi^{2}} \int_{0}^{1} \mathrm{~d} z z\left[\ln \left[1+3 \alpha_{s}^{T N P}(z)\right]-\frac{3}{4} \alpha_{s}^{T N P}(z)\right] .
$$

Let us emphasize that in order to factorize the scale dependence in the effective potential it is necessary to choose the fixed scale, like $q_{e f f}^{2}$, and not the scale which can be varied, for example like the mass gap which can go to zero in order to recover the PT limit (see section below). Eqs. (16) and (17) are the main subject of our consideration in what follows. It is worth emphasizing once more that these expressions are general ones in order to correctly calculate the Bag constant from first principles in any model gluon propagator. The only problem remaining to solve is to choose such TNP effective charge $\alpha_{s}^{T N P}(z)$ which, first of all should not explicitly depend on the gauge-fixing parameter $\xi$. At the same time, the implicit gauge dependence is not a problem. Such kind of the dependence is unavoidable in quantum or classical gauge theories, since the fields themselves are gauge-dependent [1, 2]. For the different TNP effective charges $\alpha_{s}^{T N P}(z)$ one gets different numerical results. That is why the choice for its explicit expression (ansatz) should be physically and mathematically well justified (see below).

In this connection, let us remind that the gluon Schwinger-Dyson (SD) equation is highly non-linear one, and it has a very complicated mathematical structure, so there is no hope for an exact solutions, the number of which is not even fixed [1. This means that the number of independent solutions, obtained under specific truncation/approximation schemes and gauges, is not fixed a priori as well. From the very beginning they should be considered on equal footing. 


\section{Confining effective charge}

Let us choose the TNP effective charge as follows:

$$
\alpha_{s}^{T N P}\left(q^{2}\right) \quad \longrightarrow \quad \alpha_{s}^{I N P}\left(q^{2}\right)=\frac{\Delta^{2}}{q^{2}},
$$

where the superscript "INP" stands for the intrinsically NP effective charge (for a such replacement see remarks below). Here $\Delta^{2} \equiv \Delta_{J W}^{2}$ is the so-called Jaffe-Witten (JW) mass gap, which is responsible for the large-scale structure of the QCD vacuum, and thus for its INP dynamics [24]. Let us note, that how the mass gap appears in QCD has been explicitly shown in our recent work in Ref. [25].

- The gauge independence is obvious, i.e., it does not depend explicitly on the gauge choice, since the mass gap is already renormalized, and hence it is a finite quantity.

- It satisfies the Wilson criterion of confinement - area law for heavy quarks [26, 27] or, equivalently, leads to the linear rising potential between heavy quarks [28, 29] in continuous QCD, "seen" also by lattice QCD [30, 31]. In this connection a few remarks are in order. In the case of heavy quarks the response of the vacuum can be neglected, and therefore the interaction between them and gluons effectively becomes point-pike. Just this makes it possible to describe confinement of heavy quarks in terms of the linear rising potential, derived on the basis of the expression (18). For the light quarks the response of the vacuum cannot be neglected. The corresponding quark-gluon vertex is not point-like, and therefore there is no way to analyze confinement of light quarks in terms of the linear rising potential. However, the expression (18) can be still used for the solution of the SD equation for the quark propagator together with the corresponding Slavnov-Taylor (ST) identity for the vertex [32]. Confinement of light quarks is due to the analytical properties of the corresponding Green's functions (unlike the electron propagator, the quark propagator should have no imaginary part). This is a principle difference in the description of confinement for light and heavy quarks.

- The functional dependence in the confining expression (18) is, of course, the same for the YM fields and the full QCD. The dependence on the number of flavors can appear only in the mass gap.

- It is exactly defined, since in the formal PT limit $\left(\Delta^{2}=0\right)$ the INP effective charge (18) vanishes, and hence the bag constant itself.

- It is uniquely defined as well. In order to show this explicitly, let us assume that it can be replaced by some arbitrary function as follows:

$$
\alpha_{s}^{I N P}\left(q^{2} ; \Delta^{2}\right) \longrightarrow \frac{\Delta^{2}}{q^{2}} \times f\left(q^{2} ; \Delta^{2}\right),
$$

where $f\left(q^{2} ; \Delta^{2}\right)$ is the dimensionless arbitrary function, which is regular at zero in order not to change confining properties of the INP effective charge (18). In this case it can be expand in Taylor series around small $q^{2}$, so one obtains 
$f\left(q^{2} ; \Delta^{2}\right)=f(0)+\left(q^{2} / M^{2}\right) f^{\prime}\left(q^{2} ; \Delta^{2}\right)+\ldots$, where $M^{2}$ is some auxiliary mass squared. Then the INP effective charge in Eq. (19) becomes

$$
\alpha_{s}^{I N P}\left(q^{2} ; \Delta^{2}\right)=\frac{\Delta^{2}}{q^{2}} f(0)+\frac{\Delta^{2}}{M^{2}} f^{\prime}\left(q^{2} ; \Delta^{2}\right)+\ldots,
$$

and substituting this into the general decomposition (7), one finally obtains

$$
\alpha_{s}\left(q^{2} ; \Delta^{2}\right)=\alpha_{s}^{I N P}\left(q^{2} ; \Delta^{2}\right)+\alpha_{s}^{P T}\left(q^{2}\right)=\frac{\Delta^{2}}{q^{2}}+\alpha_{s}^{P T}\left(q^{2} ; \Delta^{2}\right),
$$

where not loosing generality we include the finite number $f(0)$ into the mass gap, and retaining the same notation, for simplicity. The uniqueness is achieved at the expense of the PT effective charge, which now becomes regularly dependent on the mass gap (compare with the expression (9)). Evidently, the uniqueness is due to the singular at origin structure of the INP effective charge in Eq. (18). In Ref. [33] it has been explicitly shown that the TNP part of the full gluon propagator as a function of the mass gap contains a regular at origin term as well. That is why it is not uniquely separated from the PT gluon propagator which effective charge is always regular at origin. We distinguish between the INP and the PT effective charges not only by the explicit presence of the mass gap, but by the character of the IR singularities as well [33]. So only after the replacement of Eq. (18) the obtained expression for the bag constant (13) becomes free of all the types of the PT contributions ('contaminations'), indeed.

- In our recent work [34] we have shown that the so-called INP gluon propagator is the purely transversal in a gauge invariant way, by construction. It exactly converges to the gluon propagator, which effective charge is in Eq. (18), after the renormalization of the mass gap is completed. For preliminary analytical investigation of such behavior see Refs. [35, 36] as well (and references therein). Thus, we consider the expression (18) not only as physically and mathematically well confirmed but as uniquely justified within the confining INP QCD [34] with its own mass gap identified with the JW mass gap for the pure YM fields (see above).

- There also exist direct lattice evidences that the zero momentum modes are enhanced in the full gluon propagator (and hence in its effective charge) [37, 38, 39, 40] (and references therein). A NP finite-size scaling technique was used in Ref. [41] to study the evolution of the running coupling in the SU(3) YM lattice theory. At low energies it is shown to grow. The chosen analytical ansatz (18) can be considered as useful functional parametrization of these lattice results, indeed, while the scale of the enhancement is taken into account by the mass gap.

- It is worth noting in advance that one of the attractive additional features of Eq. (18) is that it allows one to perform an analytical summation over the Matsubara frequencies in the generalization of the expression for the bag constant to non-zero temperatures. In this case one obtains the curve of the gluon matter pressure as a function of temperature. It and all other its derivatives (entropy and energy densities, etc.) then can be directly compared with the corresponding 
thermal lattice QCD curves [42, 43]. This will make it possible for better understanding of the thermodynamical structure of the gluon matter (work in progress and preliminary numerical results are very encouraging).

In conclusion, one may consider the expression (18) as the confining ansatz, for simplicity. However, it is worth emphasizing that only it satisfies all the necessary conditions discussed above. Let us also note that for the theoretical and numerical results, depending on the confining effective charge, see discussion in section 8 .

\section{Analytical and numerical evaluation of the bag constant}

In terms of the variable in Eq. (15) for the INP effective charge (18), one gets:

$$
\alpha_{s}^{I N P}\left(q^{2}\right)=\alpha_{s}^{I N P}(z)=\frac{z_{c}}{z}, \text { where } z=\frac{q^{2}}{q_{e f f}^{2}}, \quad \text { and } z_{c}=\frac{\Delta^{2}}{q_{e f f}^{2}},
$$

so that the dimensionless effective potential (17) becomes,

$$
\Omega_{Y M}\left(z_{c}\right)=\frac{1}{\pi^{2}} \int_{0}^{1} \mathrm{~d} z z\left[\ln \left[1+\left(3 z_{c} / z\right)\right]-\frac{3}{4} \frac{z_{c}}{z}\right] .
$$

Performing an almost trivial integration in this integral, one obtains

$$
\Omega_{Y M}\left(z_{c}\right)=\frac{1}{2 \pi^{2}} z_{c}^{2}\left[\frac{3}{2 z_{c}}+\frac{1}{z_{c}^{2}} \ln \left(1+3 z_{c}\right)-9 \ln \left(1+\frac{1}{3 z_{c}}\right)\right] .
$$

It is easy to see now that as a function of $z_{c}$, the effective potential (24) approaches zero from above as $\sim z_{c}$ at $z_{c} \rightarrow 0$ limit. At infinity $z_{c} \rightarrow \infty$ it diverges as $\sim-z_{c}$. At a fixed effective scale $q_{e f f}^{2}$ and from Eq. (22) it follows that $z_{c} \rightarrow 0$ is a correct PT regime, while $z_{c} \rightarrow \infty$ is not a physical regime, since the mass gap $\Delta^{2}$ is either finite or zero (the PT limit), i.e., it cannot be infinitely large. In other words, at a fixed effective scale one recovers the correct PT limit for the bag constant, i.e., the above-mentioned normalization condition is maintained for the bag constant, as it should be.

The nontrivial second zero of the effective potential (24) follows obviously from the condition,

$$
3 z_{c}+2 \ln \left(1+3 z_{c}\right)-18 z_{c}^{2} \ln \left(1+\left(1 / 3 z_{c}\right)\right)=0,
$$

which numerical solution is

$$
z_{c}^{0}=1.3786 .
$$

Evidently, through the relation (22) this value determines a possible upper bound for $\Delta^{2}$ and lower bound for $q_{e f f}^{2}$, since $B_{Y M} / \epsilon_{Y M}$ is always positive/negative (see Figs. 3 and (4).

At $z_{c}=0$, i.e., $\Delta^{2}=0$ the effective potential (24) vanishes identically, as it should be. From the above one can conclude that this effective potential as a function of $z_{c}$ has a maximum at some finite point, see Fig. 3. In the way how it has been introduced $z_{c}$ plays the role of the constant of integration of the effective potential though being formally a parameter of the theory. In general, by taking the first derivative of the effective 


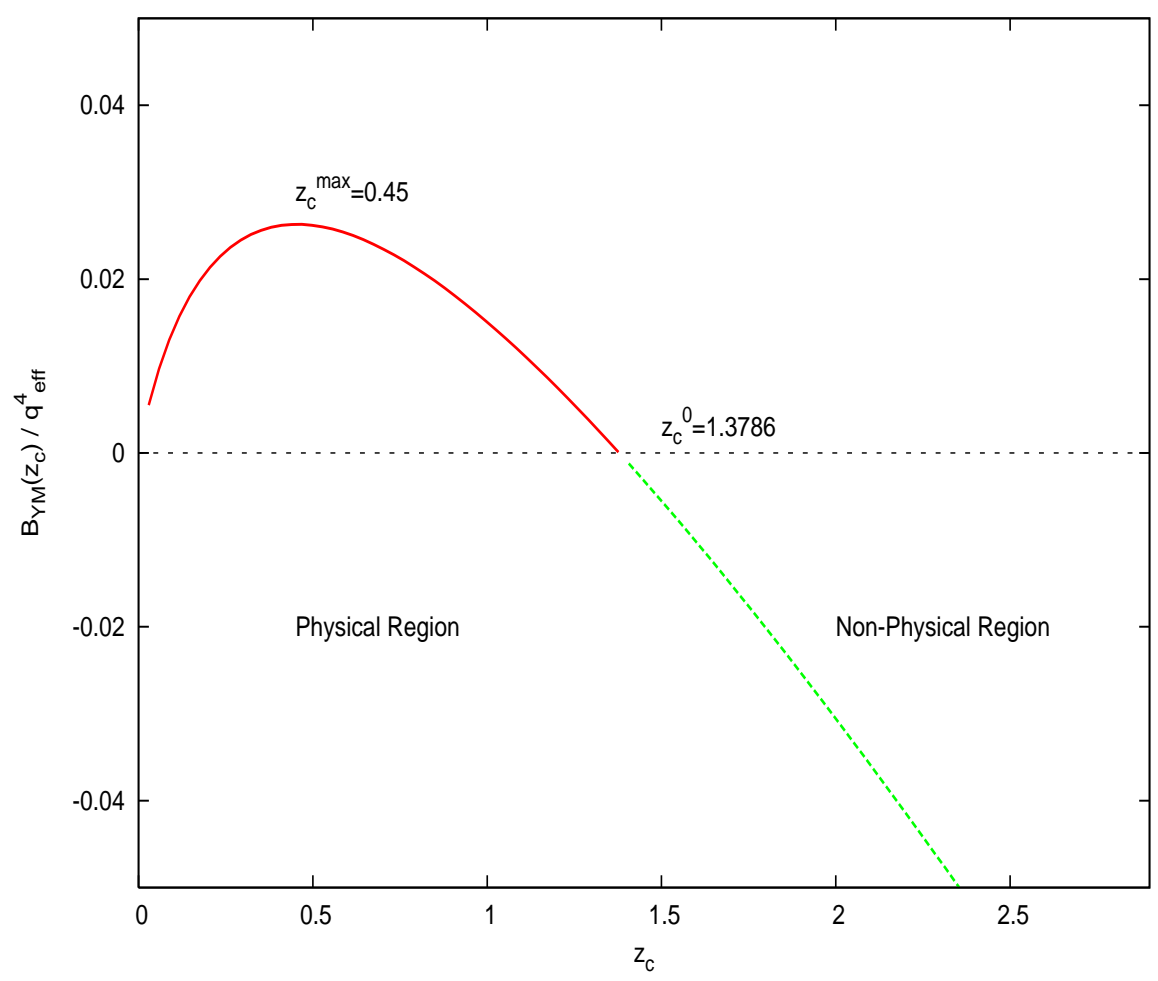

Figure 3. The $B_{Y M} / q_{e f f}^{4}$ effective potential vs. $z_{c}$. The non-physical region is $z_{c} \geq z_{c}^{0}$, since $B_{Y M}$ should be always positive. At $z_{c}=0$ the effective potential is also zero.

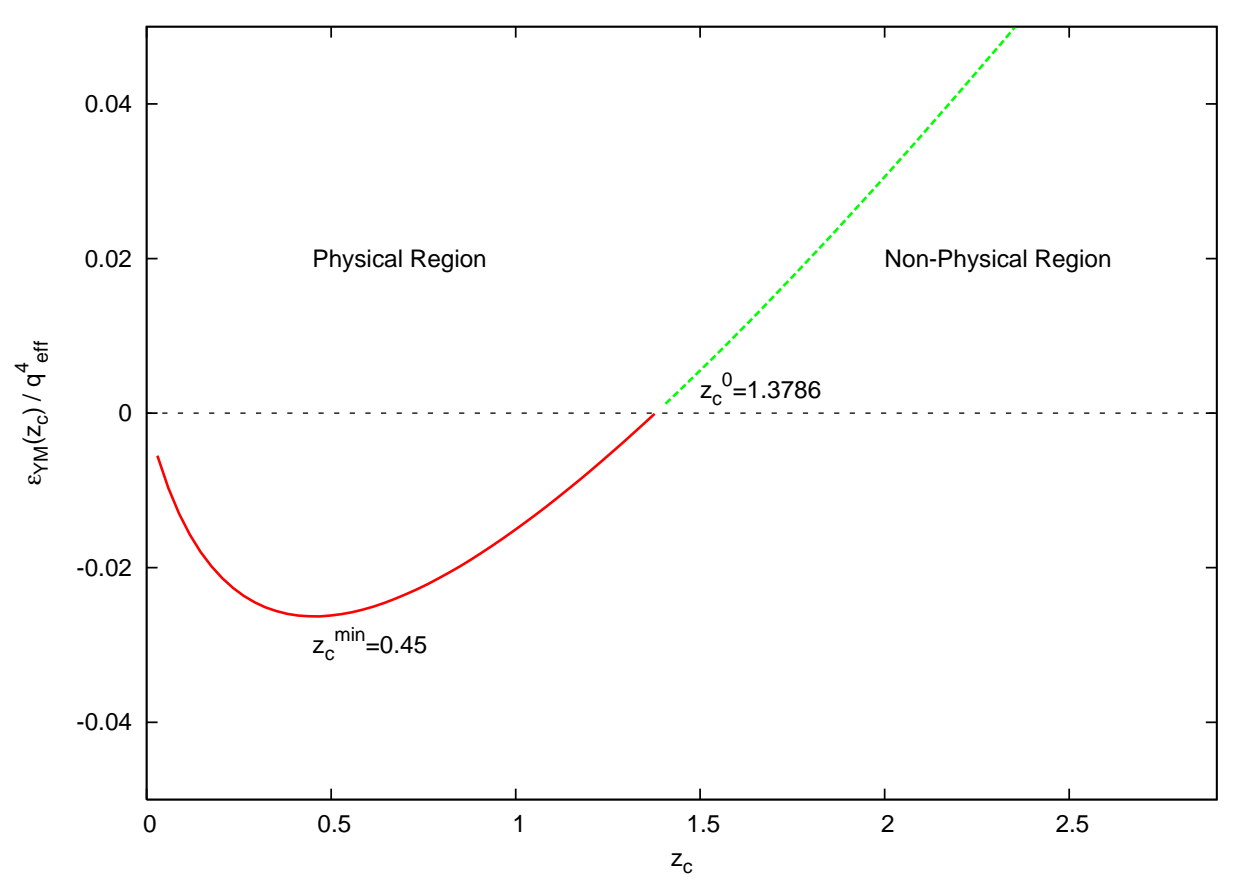

Figure 4. The $\epsilon_{Y M} / q_{\text {eff }}^{4}$ effective potential vs. $z_{c}$. The non-physical region is $z_{c} \geq z_{c}^{0}$, since $\epsilon_{Y M}$ should be always negative. At $z_{c}=0$ the effective potential is also zero. 
potential with respect to the constant of integration one recovers the corresponding equations of motion [13, 14, 15]. Requiring thus $\partial \Omega_{Y M}\left(z_{c}\right) / \partial z_{c}=0$, one obtains:

$$
z_{c}^{-1}=4 \ln \left[1+\left(1 / 3 z_{c}\right)\right]
$$

which makes it possible to fix the constant of integration of the corresponding equation of motion at maximum. Its numerical solution is

$$
z_{c}^{\max }=0.4564<1
$$

so at maximum the ratio $\Delta^{2} / q_{\text {eff }}^{2}$ is always less than one. At this point the numerical value of the effective potential (24) is

$$
\Omega_{Y M}\left(z_{c}^{\max }\right)=\frac{1}{2 \pi^{2}}\left[\frac{3}{4}\left(z_{c}^{\max }\right)-\ln \left(1+3 z_{c}^{\max }\right)\right]=0.0263 .
$$

The bag constant defined in Eq. (16), and hence the corresponding INP VED (13), as a function of $q_{e f f}^{4}$ or, equivalently, of the mass gap $\Delta^{4}$ thus becomes,

$$
B_{Y M}=-\epsilon_{Y M}=0.0263 q_{e f f}^{4}=0.1273 \times \Delta^{4},
$$

where the relation

$$
q_{e f f}^{2}=\left(z_{c}^{\max }\right)^{-1} \Delta^{2}=2.2 \Delta^{2}
$$

has been already used. It is worth noting that a maximum for the bag constant corresponds to a minimum for the INP YM VED $\epsilon_{Y M}$ (the so-called "stationary" state, see Fig. 4).

So, we have explicitly demonstrated that in the considered case the bag constant (30) is finite, positive, and it has no imaginary part, indeed. It depends only on the mass gap responsible for the INP dynamics in the QCD ground state or, equivalently, on the effective scale squared separating the NP region from the PT one.

\subsection{Scale-setting schemes and numerical results}

In order to complete the numerical calculation of the above defined bag constant all we need now is the value for the effective scale $q_{e f f}^{2}$, which separates the NP region from the PT one. Similarly, the value for a scale at which the NP effects become important, that is the mass gap $\Delta^{2}$, also allows one to achieve the same goal. If the PT regime for gluons (as well as for quarks) starts conventionally from $1 \mathrm{GeV}$, then this number is a natural choice for the effective scale. It makes it also possible to directly compare our values with the values of many phenomenological parameters calculated just at this scale (see below). We consider this value as well justified and realistic upper limit for the effective scale defined above. Thus, using further the relation (31), one gets

$$
q_{\text {eff }}^{2}=1 \mathrm{GeV}^{2} \text {, and } \Delta^{2}=0.4564 \mathrm{GeV}^{2} .
$$

Similarly, the numerical value of the mass gap $\Delta^{2}$ has been obtained from the experimental value for the pion decay constant, $F_{\pi}=93.3 \mathrm{MeV}$, by implementing a physically well-motivated scale-setting scheme [44, 45]. In fact, we approximate the 
pion decay constant in the chiral limit $F_{\pi}^{0}$ by its experimental value, since the difference between them can be a few $\mathrm{MeV}$ only. This is due to smallness of the corresponding light quark current masses. The pion decay constant is a good experimental number, since it is directly measured quantity, contrary to, for example the quark condensate or the dynamically generated quark mass. For the mass gap we have obtained the following numerical result $\Delta=0.5784 \mathrm{GeV}$, so similarly to the relations (32), one yields

$$
\Delta^{2}=0.3345 \mathrm{GeV}^{2}, \quad \text { and } q_{e f f}^{2}=0.733 \mathrm{GeV}^{2} .
$$

In what follows we will consider this value as a realistic lower limit for the effective scale. One has to conclude that we have obtained rather close numerical results for the effective scale and the mass gap, by implementing rather different scale-setting schemes. It is worth emphasizing that the effective scale (33) covers quite well not only the deep IR region but the substantial part of the intermediate one as well.

For the above-mentioned possible upper bounds for $\Delta^{2}$ and lower bounds for $q_{e f f}^{2}$ our numerical results are for the scale-setting scheme (32):

$$
\Delta^{2} \leq 1.379 \mathrm{GeV}^{2}, \quad \text { and } \quad q_{\text {eff }}^{2} \geq 0.330 \mathrm{GeV}^{2},
$$

then similarly, based on the scale-setting scheme (33):

$$
\Delta^{2} \leq 1.01 \mathrm{GeV}^{2}, \quad \text { and } \quad q_{\text {eff }}^{2} \geq 0.242 \mathrm{GeV}^{2} .
$$

Evidently, their calculated values in each scale-setting scheme satisfy the corresponding bounds.

For the bag constant (and hence for the INP YM VED) from Eq. (30), one obtains

$$
B_{Y M}=-\epsilon_{Y M}=(0.0142-0.0263) \mathrm{GeV}^{4},
$$

where the first and second numbers in brackets correspond to the numerical values given in Eqs. (32) and (33), respectively.

In conclusion, let us note that in the pure YM theory there is no way to calculate the mass gap independently of the well-motivated scale-setting scheme, that's the effective scale in this case, i.e., relations (32). The scale-setting scheme (33) is based on the numerical value of the pion decay constant in the chiral limit. So this scheme is legitimated to use here as well, since the chiral quark condensates do not contribute to the VED in this limit, as it follows from the trace anomaly relation (see next section). For further discussion on the numerical value of $B_{Y M}$ in different units see appendix B.

\section{The trace anomaly relation}

The TNP VED (and hence the bag constant) is important by itself as the main dynamical characteristic of the QCD ground state. Furthermore it assists in calculating such an important phenomenological parameter as the gluon condensate, introduced in the QCD sum rules approach to the physics of resonances [11]. The famous trace 
anomaly relation [16, 17, 18, 19] in the general case of non-zero current quark masses $m_{f}^{0}$ is

$$
\Theta_{\mu \mu}=\frac{\beta\left(\alpha_{s}\right)}{4 \alpha_{s}} G_{\mu \nu}^{a} G_{\mu \nu}^{a}+\sum_{f} m_{f}^{0} \bar{q}_{f} q_{f},
$$

where $\Theta_{\mu \mu}$ is the trace of the energy-momentum tensor and $G_{\mu \nu}^{a}$ being the gluon field strength tensor, while for the ratio $\beta\left(\alpha_{s}\right) / \alpha_{s}$ see discussion below. The trace anomaly relation which includes the anomalous dimension for the quark mass has been derived in Ref. [19], however, in our case of the pure gluon fields we can use the standard form of the trace anomaly relation (37). Sandwiching it between vacuum states and taking into account the obvious relation $\left\langle 0\left|\Theta_{\mu \mu}\right| 0\right\rangle=4 \epsilon_{t}$, one obtains

$$
4 \epsilon_{t}=\left\langle 0\left|\frac{\beta\left(\alpha_{s}\right)}{4 \alpha_{s}} G_{\mu \nu}^{a} G_{\mu \nu}^{a}\right| 0\right\rangle+\sum_{f} m_{f}^{0}\left\langle 0\left|\bar{q}_{f} q_{f}\right| 0\right\rangle .
$$

Here $\epsilon_{t}$ is the sum of all possible independent NP contributions to the VED (the total VED) and $\left\langle 0\left|\bar{q}_{f} q_{f}\right| 0\right\rangle$ is the chiral quark condensate. From this equation in the case of the pure YM fields (i.e., when the number of quark fields is zero $N_{f}=0$ ), one can get

$$
\left\langle 0\left|\frac{\beta\left(\alpha_{s}\right)}{4 \alpha_{s}} G_{\mu \nu}^{a} G_{\mu \nu}^{a}\right| 0\right\rangle=4 \epsilon_{Y M},
$$

where, evidently we saturate the total VED, $\epsilon_{t}$ by the TNP YM VED, $\epsilon_{Y M}$ defined in Eq. (13), i.e., putting $\epsilon_{t}=\epsilon_{Y M}+\ldots$ Let us note that the same result, i.e., Eq. (39), will be obtained in the chiral limit for light quarks $m_{f}^{0}=0$, for $f=1,2,3$ as well.

If confinement happens then the $\beta$ function is always in the domain of attraction (i.e., always negative) without IR stable fixed point [1]. Therefore, it is convenient to introduce the general definition of the gluon condensate not using the weak coupling limit solution to the $\beta$ function as follows:

$$
\left\langle G^{2}\right\rangle \equiv-\left\langle 0\left|\frac{\beta\left(\alpha_{s}\right)}{4 \alpha_{s}} G_{\mu \nu}^{a} G_{\mu \nu}^{a}\right| 0\right\rangle=-4 \epsilon_{Y M}=4 B_{Y M} .
$$

Thus, the above defined general gluon condensate will be always positive, as it should be. The importance of this relation is that it gives the value of the gluon condensate as a function of the bag constant whatever solution of the $\beta$ function in terms of $\alpha_{s}$ is. However, let us remind that there is a correlation between the two sides of this equation. The bag constant, correctly defined in Eq. (13), depends, in general, on the TNP effective charge $\alpha_{s}^{T N P}\left(q^{2}\right)$. On the other hand, the renormalization group equation

$$
q^{2} \frac{\mathrm{d} \alpha_{s}\left(q^{2}\right)}{\mathrm{d} q^{2}}=\beta\left(\alpha_{s}\left(q^{2}\right)\right)
$$

for the $\beta$ function gives it in terms of the corresponding effective charge. This makes it possible to determine the ratio $\left(\beta\left(\alpha_{s}\right) / \alpha_{s}\right) \equiv\left(\beta\left(\alpha_{s}\left(q^{2}\right)\right) / \alpha_{s}\left(q^{2}\right)\right)$, which appears in the left-hand-side of Eq. (40). Of course, this equation should be solved for the chosen TNP effective charge (see subsection 7.1). 
Concluding, let us only note that the quantum part of the total TNP VED at log-loop level is:

$$
\epsilon_{t}=\epsilon_{Y M}+N_{f} \epsilon_{q}
$$

where $\epsilon_{q}$ is the TNP quark skeleton loop contribution, see the corresponding skeleton loop diagram in Fig. 1. It is an order of magnitude less than $\epsilon_{Y M}$ because of much less quark degrees of freedom in the vacuum, and it is positive because of overall minus due to the quark loop. Evidently, in terms of the YM bag constant, one obtains

$$
\epsilon_{t}=-B_{Y M}\left[1-\nu N_{f}\right]
$$

where we introduce $\epsilon_{q}=\nu B_{Y M}$ and $\nu \ll 1$. So the replacement of the total bag constant by its YM counterpart only is a rather good approximation from the numerical point of view. In this connection, let us remind that in the large $N_{c}$-limit the pure gluon contribution scales as $N_{c}^{2}$, while the quark contribution scales only as $N_{c}$ [46]. However, in order to correctly calculate the bag constant in full QCD the quark part of the TNP $\operatorname{VED} \epsilon_{q}$ is also important. Let us note that it is non-zero even in the chiral limit. This part will be investigated and calculated in our subsequent paper.

\subsection{Comparison with phenomenology}

Let us show explicitly now that our numerical values for the bag constant calculated in (36) are in rather good agreement with the phenomenological values of the gluon condensate. Above we have already developed a general formalism which allows one to express the gluon condensate as a function of the bag constant. So substituting the numerical value of the bag constant into the Eq. (40), one obtains:

$$
\left\langle G^{2}\right\rangle \equiv-\left\langle 0\left|\frac{\beta\left(\alpha_{s}\right)}{4 \alpha_{s}} G_{\mu \nu}^{a} G_{\mu \nu}^{a}\right| 0\right\rangle=4 B_{Y M}=(0.0568-0.1052) \mathrm{GeV}^{4} .
$$

On the other hand, the renormalization group equation for the $\beta$ function (41) after substitution of our solution for the INP effective charge (18) yields:

$$
\beta\left(\alpha_{s}\left(q^{2}\right)\right)=-\alpha_{s}\left(q^{2}\right),
$$

as it is required for the confining theory where the $\beta$ function should be always in the domain of attraction, i.e., negative (see in Ref. [1]). The corresponding ratio as it appears in the left-hand-side of Eq. (44) is

$$
\frac{\beta\left(\alpha_{s}\right)}{\alpha_{s}} \equiv \frac{\beta\left(\alpha_{s}\left(q^{2}\right)\right)}{\alpha_{s}\left(q^{2}\right)}=-1 .
$$

Substituting further this solution into the Eq. (44), it becomes

$$
\left\langle 0\left|\frac{1}{4} G_{\mu \nu}^{a} G_{\mu \nu}^{a}\right| 0\right\rangle=4 B_{Y M}=(0.0568-0.1052) \mathrm{GeV}^{4},
$$

which means that both sides of this relation between the Bag constant and the gluon condensate have been calculated by using the same expression for the INP effective charge, and hence for the corresponding $\beta$ function. So from the numerical point of view the Bag constant and the gluon condensate are in a self-consistent dependence from 
each other, making thus the latter one free of all the types of the PT contributions. Our expression for the gluon condensate (47) allows one to recalculate any gluon condensate at any scale and any ratio, $\beta\left(\alpha_{s}\right) / \alpha_{s}$. To the gluon condensate a physical meaning can be indeed assigned as the global (average) vacuum characteristic which measures a density of the TNP gluon fields configurations in the QCD vacuum.

However, it cannot be directly compared with the phenomenological values for the standard gluon condensate estimated within different approaches [20]. The problem is that it is necessary to remember that any value at the scale as in Eq. (33) (lower bound in the right-hand-side of Eq. (47)) is to be recalculated at the $1 \mathrm{GeV}$ scale. Moreover, both values explicitly shown in Eq. (47) should be recalculated at the same ratio, as mentioned above.

In phenomenology the standard ratio of the gluon condensate and its numerical value is:

$$
G_{2}=\left\langle\frac{\alpha_{s}}{\pi} G^{2}\right\rangle=\left\langle 0\left|\frac{\alpha_{s}}{\pi} G_{\mu \nu}^{a} G_{\mu \nu}^{a}\right| 0\right\rangle \approx 0.012 \mathrm{GeV}^{4},
$$

which can be changed within a factor of $\sim 2$ [11] (let us recall that this ratio comes from the weak coupling solution for the $\beta$ function, see for example in Ref. [47]).

Thus in order to achieve the same ratio the both sides of Eq. (47) should be multiplied by $4\left(\alpha_{s} / \pi\right)$. For the numerical value of the strong fine structure constant we use $\alpha_{s}=\alpha_{s}\left(m_{Z}\right)=0.1187$ from the Particle Data Group [48]. In addition, the lower bound should be multiplied by the factor $(1 / 0.733)^{2}=1.86$, coming form the numerical value by Eq. (33). Then the recalculated gluon condensate in (47), which is denoted as $\bar{G}_{2}$, finally becomes (i.e., both numbers in Eq. (47) coincides, as it should be)

$$
\bar{G}_{2} \approx 0.016 \mathrm{GeV}^{4} \text {. }
$$

This numerical value for the gluon condensate should be compared with the numerical value coming from the phenomenology, see Eq. (48) above. This shows that all our numerical results are in good agreement with various phenomenological estimates [11, 20, taking into account that the quark contributions are approximately an order of magnitude less than the pure YM one to the full bag constant (see remarks in this section just before subsection 7.1). This confirms that our numerical values for the bag constant and hence for the gluon condensate are rather realistic ones.

\section{Conclusions}

In summary, we have formulated a general method how to calculate numerically the quantum part of the TNP YM VED (the YM bag constant, apart from the sign, by definition) in the covariant gauge QCD from first principles. For this purpose we have used the effective potential approach for composite operators to leading order [13]. It has an advantage to be directly the VED (the pressure) in the absence of external sources. The bag constant is defined as a special function of the TNP effective charge integrated out over the NP region (soft momentum region), see Eq. (13). At this stage the bag constant is colorless (color-singlet) and depends only on the transversal ("physical") 
degrees of freedom of gauge bosons. It is also free of the PT contributions by its construction. This has been achieved due to the subtractions at the fundamental level as given by Eg. (77), as well as due to all other subtractions explicitly shown in Eq. (12), when the gluon degrees of freedom were to be integrated out. Thus, our equations (16) and (17) are general ones in order to correctly calculate the bag constant as a function of any properly defined TNP effective charge.

For the concrete calculation of the bag constant we replace the TNP effective charge by its confining INP counterpart in Eq. (18), since it is exactly and uniquely separated from the PT effective charge. The INP effective charge depends regularly on the mass gap, which is responsible for the large-scale structure of the QCD ground state [24, 34]. The scale-setting schemes have been chosen by the two different ways, leading, nevertheless, to a rather close numerical results for the mass gap and hence for the effective scale. The calculated bag constant in addition, is: finite, positive, and it has no imaginary part (stable vacuum). It is also a manifestly gauge-invariant quantity (i.e., does not explicitly depend on the gauge-fixing parameter as it is required). The separation of "soft versus hard" gluon momenta is also exact because of the maximization/minimization procedure. It becomes possible since the effective potential (24) as a function of the constant of the integration $z_{c}$ and hence of the mass gap $\Delta^{2}$ has a local maximum, see Fig. 3. This also makes it possible that in the above-mentioned scale-setting schemes either the mass gap or the effective scale is only independent, since the other one is to be determined via the relation (31). In the scale-setting scheme (32) the effective scale is independent, while in the second scale-setting scheme (33) the mass gap is independent. It is worth emphasizing that the bag constant in our approach is not simply the difference between the PT and NP VEDs, which is finite, colorless and manifestly gauge-invariant, etc. It is the energy density (apart from the sign) of the system of stable configurations of the purely transversal quantum virtual fields with the enhanced low-frequency components/large scale amplitudes due to the $N L$ interaction of massless gluon modes, and which is being at "stationary state", i.e., being in the state with the minimum of energy, see Fig. 4.

In order to compare our numerical results with phenomenology we develop a general formalism which makes it possible to relate the bag constant to the gluon condensate in a unique and self-consistent way. In other words, the gluon condensate is defined and calculated at the same effective charge, which has been chosen for the calculation of the bag constant. For this purpose we use the trace anomaly relation without applying to the weak coupling solution for the corresponding $\beta$ function. In its turn, it is a solution of the corresponding renormalization group equation for the effective charge Eq. (41). Our numerical results turned out to be in good agreement with phenomenological values of the gluon condensate calculated and estimated within different approaches and methods [11, 20].

It is instructive to briefly summarize our theoretical and numerical results for the bag constant in general and our specific ways: 
General properties of the bag constant determined by Eqs. (16)-(17) are:

- colorless (color-singlet);

- electrically neutral;

- transversal, i.e., depending only on "physical" degrees of freedom of gauge bosons;

- free of the PT contributions ('contaminations').

Results, depending on the confining effective charge Eq. (18) are:

- the explicit gauge invariance;

- uniqueness, i.e., it is free of all the types of the PT contributions now;

- finiteness;

- positiveness;

- no imaginary part (stable vacuum);

- existence of the stationary state for the corresponding YM energy density (negative pressure, see Fig. 4);

- the final dependence on the mass gap only;

- a good numerical agreement with phenomenology.

The above remarkable features all together are unique. Apparently, it is due to the confining expression (18) and the correct determination of the bag constant itself in this investigation. It has been made in accordance with its modern definition as the difference between the PT and the NP VEDs [9, 10, 11, 12].

Our method can be generalized on the multi-loop skeleton contributions to the effective potential approach for composite operators, as well as to take into account the quark degrees of freedom, as plotted in Fig. 1. These terms, however, will produce numerical contributions an order of magnitude less, at least, in comparison with the leading log-loop level gluon term given by Eq. (11). What is necessary indeed, is to be able to extract the finite part of the TNP VED in a self-consistent and manifestly gauge-invariant ways. This is provided by our method which thus can be applied to any QCD vacuum quantum and classical models at any gauge (covariant or non-covariant). It may serve as a test of them, providing an exact criterion for the separation "stable versus unstable" vacua. Using our method we have already shown that the vacuum of classical dual Abelian Higgs model with string and without string contributions is unstable against quantum corrections [49, 50].

It would be also interesting to apply our general equations (16) and (17) in order to calculate the bag constant within the recently obtained analytical results for the gluon propagator in Refs. [51, 52, 53, 54, 55, 56, 57, 58]. The general formalism developed in our paper is aimed first of all at the analytical calculations of the bag constant (or the vacuum energy density) in any model gluon propagator in continuous QCD. However, as mentioned above the chosen ansatz (18) can be considered as useful parametrization of the corresponding lattice results. In this way our formalism can be extended to the 
lattice calculations as well. Choosing an appropriate parametrization of any lattice result for the gluon propagator (there is a lot of recent lattice data [59, 60, 61, 62] and references therein), one then can substitute it into our analytical expressions (16)-(17). Such a combination of the lattice and analytical calculations can be rather effective indeed, in order to understand what is the physics behind the lattice numbers and curves. On the other hand, all the analytical expressions and calculations will be put on solid numerical ground provided by the lattice simulations. So there is no doubt that the analytical and lattice calculations should not exclude each other, but contrary they should complement each other. All these possible developments are, of course, beyond the scope of the present investigation, and they have to be done elsewhere.

\section{Acknowledgments}

Support in part by HAS-JINR and Hungarian OTKA-TO47050, NK62044 and IN71374 (P. Lévai), PD73596 (GGB) is to be acknowledged. We are grateful to J. Nyiri for constant support and help. We would like also to thank M. Vasúth for help in the numerical calculations and Á. Lukács for useful discussion. This paper was partially supported by the János Bolyai Researc Scholarship of the HAS.

\section{Appendix A. The general role of ghosts}

Let us begin with recalling that due to the above-mentioned normalization condition in the initial Eq. (1), its elaborated counterpart in Eq. (6) depends only on the transversal ("physical") component of the full gluon propagator. So there is no need for ghosts to cancel its longitudinal (unphysical) component, indeed. However, it is instructive to discuss the role of ghosts in general, and to clearly show that their explicit introduction leads to the same result for the bag constant, in particular.

Following Ref. [13], the effective potential at the same log-loop order for the ghost degrees of freedom analytically is:

$$
V(G)=-i \int \frac{\mathrm{d}^{4} k}{(2 \pi)^{4}} \operatorname{Tr}\left\{\ln \left(G_{0}^{-1} G\right)-\left(G_{0}^{-1} G\right)+1\right\},
$$

where $G \equiv G(k)=i / k^{2}\left(1+b\left(k^{2}\right)\right)$ is the full ghost propagator, where $b\left(k^{2}\right)$ is the ghost self-energy, while $G_{0} \equiv G_{0}(k)=i / k^{2}$ is its free PT counterpart. Trace over color group indices is assumed. Evidently, the effective potential is normalized to $V\left(G_{0}\right)=0$ in the same way as the gluon part in Eq. (11). Substituting these expressions into the ghost term (A.1) and again doing some algebra in four-dimensional Euclidean space, one formally obtains that $V(G)=\epsilon_{g h}=\int \mathrm{d} k^{2} f\left(b\left(k^{2}\right)\right)$. This, in general, divergent constant contribution should be of course, regularized in order to assign to it a mathematical meaning. So the explicit functional dependence of the ghost propagator/self-energy on its argument is not important, since within the effective potential approach to calculate the VED it is always only constant. We have to sum up all the contributions for the 
pure YM fields at the same skeleton log-loop order. The relation given by Eq. (10) then should look like as:

$$
\begin{aligned}
\epsilon_{g}+\epsilon_{g h}= & -\frac{1}{\pi^{2}} \int_{0}^{q_{e f f}^{2}} \mathrm{~d} q^{2} q^{2}\left[\ln \left[1+3 d^{T N P}\left(q^{2}\right)\right]-\frac{3}{4} d^{T N P}\left(q^{2}\right)\right]+ \\
& +\epsilon_{P T}+\epsilon_{P T}^{\prime}+\epsilon_{g h} .
\end{aligned}
$$

It is worth emphasizing that, the right-hand-side of this relation may still suffer from unphysical singularities by the integral in Eq (9), defining $\epsilon_{P T}$. The problem is that the PT effective charge, $d^{P T}\left(q^{2}\right)$, which is responsible for AF in QCD at large $q^{2}$ (see, for example our paper [34]), may have, in general, unphysical singularities below the scale $\Lambda_{Q C D}^{2}$, since in the integral (9) the integration is from zero to infinity. In addition, as mentioned above the integral (11), defining $\epsilon_{P T}^{\prime}$, may be still divergent. Thus the left-hand-side of the relation (A.2) is formal one, indeed. It suffers from various types of unphysical singularities which may appear in its right-hand-side. In order to get a physically meaningful expression, one has to remove the two integrals (9) and (11) from Eq. (6). This is to be done with the help of a ghost term by imposing the following condition of cancelation of unwanted terms in the most general form: $\epsilon_{P T}+\epsilon_{P T}^{\prime}+\epsilon_{g h}=0$. This condition can be always fulfilled, since it is a relation between three different (unknown in general) regularized constants. Then the relation (A.2) thus becomes:

$$
\begin{aligned}
\epsilon_{Y M} & =\epsilon_{g}-\epsilon_{P T}-\epsilon_{P T}^{\prime} \\
& =-\frac{1}{\pi^{2}} \int_{0}^{q_{e f f}^{2}} \mathrm{~d} q^{2} q^{2}\left[\ln \left[1+3 d^{T N P}\left(q^{2}\right)\right]-\frac{3}{4} d^{T N P}\left(q^{2}\right)\right],
\end{aligned}
$$

in complete agreement with the relation (12), and hence with the definition of the bag constant (13), as it should be. So the TNP gluon contribution to the VED has been determined by subtracting unwanted terms by means of the ghost contribution. Evidently, the subtracted terms are of no importance, while a ghost term plays no explicit role for further consideration.

In QCD the general role of ghost degrees of freedom is to cancel all the unphysical degrees of freedom of gauge bosons [1, 63, maintaining thus unitarity of the $S$-matrix. This is the main reason why they should be taken into account together with gluons always. This means that nothing should explicitly depend on them after the abovementioned cancelation is performed. One of the main purposes of their introduction is to exclude the longitudinal (unphysical) component of the gluon propagator in every order of the PT, thus going beyond it and thus being a general one, indeed. If there is no need to cancel the longitudinal component of gauge boson propagators, then they should be used to eliminate the unphysical singularities of gauge bosons below the QCD asymptotic scale (as it was described above), or some other ones which may be inevitably present in any solution/ansatz for the full gluon propagator. If one knows the ghost propagator exactly, then the above-mentioned cancelation of unphysical singularities of gauge bosons should proceed automatically, as usual in the PT calculus (if, of course, 
all calculations are correct). For such an exact cancelation of the longitudinal part of the gluon propagator by the free PT ghost propagator in lower order of the PT see, for example Ref. [63]. But if it is not known exactly or known approximately (depending on the truncation/approximation scheme), as usual in the NP calculus then nevertheless, one has to impose the corresponding condition of cancelation in order to fulfill their general role. This just has been done above. Thus our subtraction scheme is in agreement with the general interpretation of ghosts to cancel all the unphysical degrees of freedom of gauge bosons [1, 63. So by themselves the ghosts cannot change the truly NP dynamics of QCD, associated with the transversal component of the full gluon propagator in Eq. (2) and described by its Lorentz structure or, equivalently, by its effective charge (see Ref. [25] as well).

Whatever solution(s) for the full gluon propagator obtained by lattice QCD [59, 60, 61, 62] (and references therein) and by the analytical approach based on the corresponding SD system of equations [51, 52, 53, 54, 55, 56, 57, 58] (and references therein) might be (smooth, singular, massive, etc.), it, however, should not undermine the above-mentioned general job of ghosts. It is worth emphasizing that by no coincidence in all the papers cited above the transversal Landau gauge has been chosen by hand from the very beginning. So there is no and cannot be the explicit dependence on the ghost degrees of freedom in any expressions for the physical quantities, in general, and in the expression for the bag constant, in particular. In this connection, let us remind that the confining effective charge (18) is the effective charge of the relevant gluon propagator, which becomes the purely transversal in a gauge invariant way, by construction [33, 34].

Nevertheless, this does not mean that we need no ghosts at all. First of all, we need them in the higher orders of the two-particle irreducible vacuum graphs in the skeleton loop expansion of the effective potential [13], since for them the simple normalization of the free PT vacuum to zero does not work. So the cancelation of unphysical gluon modes should proceed with the help of the ghost degrees of freedom, as it was described in this appendix above. It is necessary to understand that the transversality of the gluon propagator in the Landau gauge in order to correctly treat the PT QCD Green's functions without ghosts is not enough to insure unitarity of the $S$-matrix in QCD. The whole machinery of all the ST identities and the corresponding SD equations is still needed in order to insure the unitarity cancelations even in the Landau gauge.

For example, the quark ST identity, contains the so-called ghost-quark scattering kernel explicitly [1]. This kernel still makes an important contribution to the identity even if the gluon propagator is transversal [64, 65]. Omitting ghosts at all in this identity, one will lose an important piece of information on the quark degrees of freedom themselves. As a result, any solution of the quark SD equation will suffer from unphysical singularities in the complex momentum plane. The problem is that via the quark-gluon vertex this equation will crucially depend on the term which comes from the identity even if the gluon propagator is transversal. The completely NP analysis of this identity on the basis of the double pole structure of the full gluon propagator in the IR, Eq. (18), has 
been made in our earlier papers [66, 67]. We have derived the corresponding expression for the quark-gluon vertex following Ref. 68] only in more sophisticated way (see Ref. [32] as well). We will take this result into account when we will directly calculate the confining quark contribution to the bag constant, as mentioned in section 7 just before subsection 7.1 .

\section{Appendix B. Numerical values for $B_{Y M}$ in different units}

In order to show explicitly what magnitude of numbers we are dealing with, let us present our numerical value for the bag constant given by Eq. (36) in different units, namely:

$$
\begin{aligned}
B_{Y M}=-\epsilon_{Y M} & =(0.0142-0.0263) \mathrm{GeV}^{4} \\
& =(1.84-3.4) \mathrm{GeV} / \mathrm{fm}^{3} \\
& =(1.84-3.4) \times 10^{39} \mathrm{GeV} / \mathrm{cm}^{3} .
\end{aligned}
$$

This is a huge amount of energy stored in one $\mathrm{cm}^{3}$ of the QCD vacuum even in "Godgiven" units $\hbar=c=1$. Using the number of different conversion factors (see, for example Ref. [63] or the particle data group [48]) the bag constant can be expressed in different systems of units (SI, CGS, etc.).

Taking further into account that

$$
1 \mathrm{GeV}=1.6 \times 10^{10} \mathrm{~J}=4.45 \times 10^{-23} \mathrm{GWh},
$$

from Eq. (B.1) one finally gets $\left(1 \mathrm{~W}=10^{-3} \mathrm{~kW}=10^{-6} \mathrm{MW}=10^{-9} \mathrm{GW}\right)$

$$
B_{Y M}=(8.2-15) \times 10^{16} \mathrm{GWh} / \mathrm{cm}^{3}
$$

or, equivalently,

$$
E_{Y M}=B_{Y M} \mathrm{~cm}^{3}=(8.2-15) \times 10^{16} \mathrm{GWh} \sim 10^{17} \mathrm{GWh}
$$

in familiar units of watt-hour (Wh). Let us note that if one puts the effective scale squared as small as realistically possible $q_{\text {eff }}^{2}=0.242 \mathrm{GeV}^{2}$ (see Eq. (35) ), then the previous number will be only slightly changed, namely $E_{Y M}=B_{Y M} \mathrm{~cm}^{3}=$ $(4.8-8.7) \times 10^{15}$ GWh. So both numbers still indicate a huge amount of the bag energy $E_{Y M}$ stored in one $\mathrm{cm}^{3}$ of the QCD vacuum.

It is especially interesting to compare these numbers with the total production of primary energy of the $25 \mathrm{EU}$ countries in year 2004 which was [69] (see also Ref. [70])

$$
E_{t} \sim 10.2 \mathrm{PWh}=10.2 \times 10^{6} \mathrm{GWh} \sim 10^{7} \mathrm{GWh},
$$

where $1 \mathrm{PWh}=1$ Petawatt-hour. Approximately 1/3 of this energy was produced by nuclear power plants [69, 70]. The huge difference between the numbers in Eqs. (B.4) and (B.5) is very impressive and leads to some interesting still speculative but already possible discussion in appendix D below and in our preliminary work [71]. 


\section{Appendix C. Contribution of $B_{Y M}$ to the dark energy problem}

Apparently, our bag constant (B.1) may also contribute to the so-called dark energy density [72]. At least, from the qualitative point of view it satisfies almost all the criteria necessary for the dark energy/matter candidate (see here section 8 and discussions in Refs. [72, 73]). From the quantitative numerical point of view it is also much better than the estimate from the Higgs field's contribution to the VED, which is about [74, 75]

$$
\varrho_{H} \sim 10^{8} \mathrm{GeV}^{4} .
$$

In this notation our value (B.1) is about

$$
\varrho_{\text {our }} \sim 10^{-2} \mathrm{GeV}^{4} .
$$

The observed VED is very small indeed, namely

$$
\varrho_{v a c} \sim 10^{-46} \mathrm{GeV}^{4},
$$

see Refs. [74, 175, 76]. So relatively to the value inferred from the cosmological constant (i.e., the above-mentioned observed VED)

$$
\varrho_{H} / \varrho_{v a c} \sim 10^{54},
$$

while our is

$$
\varrho_{\text {our }} / \varrho_{\text {vac }} \sim 10^{44},
$$

i.e, some 10 orders of magnitude better, which is expected from the direct comparison of the estimate (C.1) with our value (C.2).

Let us note that calculating at the Plank length scale [48], we will obtain the same ratio, as it should be. From Eq. (B.1) it follows that

$$
\varrho_{\text {our }} \sim 10^{39} \mathrm{GeV} / \mathrm{cm}^{3}=10^{-60} \mathrm{GeV} / L_{p}^{3},
$$

where we used $\mathrm{cm}=10^{33} L_{p}$ and $L_{p}$ denotes the above-mentioned Plank length [48]. In this units the observed VED is

$$
\varrho_{\text {vac }} \sim 10^{-46} \mathrm{GeV}^{4} \sim 10^{-5} \mathrm{GeV} / \mathrm{cm}^{3}=10^{-104} \mathrm{GeV} / L_{p}^{3},
$$

so that the ratio between (C.6) and (C.7) becomes again (C.5), indeed. Of course, the ratio (C.5) still remains very large, but it is much better than the ratio (C.4), as emphasized above. Other possibility how QCD can be related to the dark energy puzzle has been described in Ref. [77] (and references therein).

Concluding, the vacuum for which the value (C.3) has been measured should not be mixed with the vacuum of any quantum field gauge theory. For the former one its energy is always positive (i.e., above zero), so the vacuum is simply treated as empty space. The energy of the latter one is always negative (i.e., below zero), and it is full of any kind of quantum excitations, fluctuations, etc. However, the QCD bag constant is always positive, finite, gauge-invariant, etc. (if it has been correctly defined and calculated like in this work). That is the primary reason why we can compare our value (C.2) and the estimate (C.1) with (C.3). 


\section{Appendix D. Energy from the QCD vacuum}

The Lamb shift and the Casimir effect are probably the two most famous experimental evidences of zero-point energy fluctuations in the vacuum of Quantum Electrodynamics (QED) [78, 79, 80, 81]. Both effects are rather weak, since the QED vacuum is mainly PT by origin, character and magnitude (the corresponding fine structure constant is weak). However, even in this case attempts have been already made to exploit the Casimir effect in order to "observe" the negative energy and related affects 80. and even to release energy from the vacuum (see, for example Refs. [82, 83] and references in the above-mentioned reviews [78, 79]). In Ref. [84] by investigating the thermodynamical properties of the quantum vacuum it has been concluded that no energy can be extracted cyclically from the vacuum (see, however Ref. [78] and references therein). Let us also note that in QED the photon propagator always remains PT even "dressed" [25, 34, 85, 86]. So formally we can define the bag constant in this theory as $B_{Q E D}=V E D^{0}-V E D=-V E D>0$, since $V E D^{0} \equiv V E D\left(D_{0}\right)=0$ in the effective potential approach to leading order [13]. It would be interesting to perform such a calculation, which will give one a correct finite value of the VED in QED, if, or course, the above proposed definition of the QED bag constant makes sense. But it is beyond the scope of the present investigation, and should be done elsewhere.

Since the QCD fine structure constant is strong, the idea to exploit the QCD vacuum in order to extract energy from it seems to be more attractive. However, before discussing the ways how to extract, it is necessary to discuss which minimum/maximum amount of energy at all can be released in a single cycle. Who thinks that it is too early to discuss such kind of topic (though we do not think so) may entirely skip this appendix.

The bag constant calculated here is a manifestly gauge-invariant, real and colorless (color-singlet) quantity, i.e., it can be considered as a physical quantity. In fact, in this paper we have formulated a renormalization program to make the bag constant or, equivalently, the bag pressure finite and satisfying all other necessary requirements (see section 8 above). The key elements of this program were the necessary subtractions at all levels. Moreover, one of its attractive features, as emphasized above, is that it is the energy density of the purely transversal virtual gluon field configurations which are not only stable (no imaginary part), but are being in the stationary state as well, i.e., in the state with the minimum of energy (see Fig. 4). That is why it makes sense to discuss the "releasing" of the bag constant from the vacuum, more precisely the bag energy (B.4).

From the quantum statistical mechanics point of view, the energy is nothing but the pressure multiplied by the volume $V$ in the infinite-volume limit [87]. So the vacuum energy $E_{v a c}$ in terms of the bag constant is and in $\mathrm{GeV}$ units it diverges as follows:

$$
E_{v a c}=-B_{Y M} V=-E_{Y M} \frac{V}{\mathrm{~cm}^{3}} \sim-\lambda^{3}, \quad \lambda \rightarrow \infty,
$$

since $V / \mathrm{cm}^{3} \sim \lambda^{3}$ always when the dimensionless UV cutoff $\lambda$ goes to infinity. Evidently, in deriving Eq. (D.1) we use the general relation $E_{Y M}=B_{Y M} \mathrm{~cm}^{3}$, which is valid in 
any units for energy (see appendix B above).

Let us imagine now that we can release the finite portion $E_{Y M}$ (B.4) from the vacuum in $k$ different places (different "vacuum energy releasing facilities" (VERF)). It can be done by $n_{m}$ times in each place, where $m=1,2,3 \ldots k$. Then the releasing energy $E_{r}$ becomes

$$
E_{r}=E_{Y M} \sum_{m=1}^{k} n_{m}
$$

The ideal case (which, however, will never be achieved) is when we could extract a finite portion of the energy an infinite number of times and in an infinite number of places. So the releasing energy (D.2) might be divergent as follows:

$$
E_{r}=E_{Y M} \times \lim _{\left(k, n_{m}\right) \rightarrow \infty} \sum_{m=1}^{k} n_{m} \sim \lambda^{2}, \quad \lambda \rightarrow \infty,
$$

since the sum over $m$ diverges quadratically in the $\lambda \rightarrow \infty$ limit, and $k \sim \lambda, n_{m} \sim \lambda$ in this case. The difference between the vacuum energy (D.1) and the releasing energy (D.3) which is nothing but the remaining in the vacuum energy $E_{R}$ becomes

$$
E_{R}=E_{v a c}-E_{r}=E_{v a c}[1+O(1 / \lambda)], \quad \lambda \rightarrow \infty,
$$

i.e., the QCD vacuum is an infinite and permanent reservoir of energy. The situation is even "better" if one takes into account the PT contributions to the vacuum energy (in this case the convergence becomes of the order $O\left(1 / \lambda^{2}\right)$ in Eq. (D.4), see our preliminary work in Ref. [71]).

That's the vacuum energy is badly divergent is not a mathematical problem. This reflects an universal reality. Vacuum is everywhere and it always exists. Quite possible that our Universe in general and our real word in particular is only its special type of excitation due to the Big Bang. As underlined above, the vacuum is an infinite and hence a permanent source of energy. The only problem is how to release the finite portion the bag energy (B.4) and whether it will be profitable or not by introducing some type of cyclic process. However, due to huge difference between the two numbers (B.4) and (B.5) such a cyclically profitable process may be realistic. "Perpetuum mobile" does not exist, but "perpetuum source" of energy does exist, and it is the QCD ground state.

\section{References}

[1] W. Marciano, H. Pagels, Phys. Rep. C 36 (1978) 137.

[2] V.A. Rubakov, Classical Gauge Fields (Editorial YRSS, Moscaw, 1999).

[3] Confinement, Duality, and Nonperturbative Aspects of QCD, edited by by P. van Baal, NATO ASI, Series B: Physics, vol. 368 (Plenum, New York, 1997).

[4] Non-Perturbative QCD, Structure of the QCD Vacuum, edited by K-I. Aoki, O. Miymura, and T. Suzuki [Prog. Theor. Phys. Suppl. 131 (1998) 1].

[5] V.N. Gribov, Gauge Theories and Quark Confinement (PHASIS, Moscow, 2002).

[6] Y.L. Dokshitzer, D.E. Kharzeev, Ann. Rev. Nucl. Part. Sci. 54 (2004) 487; arXiv:hep-ph/0404216. 
[7] A. Chodos et al., Phys. Rev. D 9 (1974) 3471.

[8] T. DeGrand et al., Phys. Rev. D 12 (1975) 2060.

[9] E.V. Shuryak, Phys. Rep. 115 (1984) 151.

[10] M.S. Chanowitz, S. Sharpe, Nucl. Phys. B 222 (1983) 211.

[11] M.A. Shifman, A.I. Vainshtein, V.I. Zakharov, Nucl. Phys. B 147 (1979) 385.

[12] V.A. Novikov, M.A. Shifman, A.J. Vainshtein and V.A. Zakharov, Nucl. Phys. B 191 (1981) 301.

[13] J.M. Cornwall, R. Jackiw, E. Tomboulis, Phys. Rev. D 10 (1974) 2428.

[14] A. Barducci et al., Phys. Rev. D 38 (1988) 238.

[15] R.W. Haymaker, Riv. Nuovo Cim. 14 (1991) 1-89.

[16] R.J. Crewther, Phys. Rev. Lett. 28 (1972) 1421.

[17] M.S. Chanowitz, J. Ellis, Phys. Rev. D 7 (1973) 2490.

[18] J.C. Collins, A. Duncan, S.D. Joglecar, Phys. Rev. D 16 (1977) 438.

[19] B. Grinstein, L. Randall, Phys. Lett. B 217 (1989) 335.

[20] S. Narison, Phys. Lett. B 387 (1996) 162.

[21] P. Castorina, So-Y. Pi, Phys. Rev. D 31 (1985) 411.

[22] M. Baker, J.S. Ball, F. Zachariasen, Phys. Rev. D 37 (1988) 1036.

[23] M.N. Chernodub, M.I. Polikarpov, V.I. Zakharov, Phys. Lett. B 457 (1999) 147; arXiv:hep-ph/9903272

[24] A. Jaffe, E. Witten, Yang-Mills Existence and Mass Gap, http://www.claymath.org/prize-problems/, http://www.arthurjaffe.com

[25] V. Gogokhia, Int. J. Theor. Phys. 48 (2009) 3061; arXiv:0806.0247 [hep-th], [hep-ph].

[26] K.B. Wilson, Phys. Rev. D 10 (1974) 2445.

[27] M. Bander, Phys. Rep. 75 (1981) 205.

[28] A.I. Alekseev and B.A. Arbuzov, Physics of Atomic Nuclei 61 (1998) 264.

[29] A.I. Alekseev, hep-ph/0503242.

[30] K.D. Born et al., Phys. Lett. B 329 (1994) 325.

[31] V.M. Miller et al., Phys. Lett. B 335 (1994) 71.

[32] V. Gogokhia, arXiv:0704.1745 [hep-ph]; arXiv:0704.3189 [hep-ph].

[33] V. Gogokhia, Int. J. Theor. Phys. 48 (2009) 3470; arXiv:0904.2266 [hep-ph], [hep-th].

[34] V. Gogokhia, Int. J. Theor. Phys. 48 (2009) 3449; arXiv:0907.0082 [hep-ph], [hep-th].

[35] V. Gogohia, Phys. Lett. B 584 (2004) 225.

[36] V. Gogohia, Phys. Lett. B 618 (2005) 103.

[37] C. Michael, Nucl. Phys. B (Proc. Suppl.) 42 (1995) 147.

[38] G. Damm, W. Kerler, V. K. Mitrjushkin, Phys. Lett. B 443 (1998) 88.

[39] G. Burgio, F. Di Renzo, C. Parrinello, C. Pittori, Nucl. Phys. B (Proc. Suppl.) 73 (1999) 623.

[40] G. Burgio, F. Di Renzo, G. Marchesini, E. Onofri, Nucl. Phys. B (Proc. Suppl.) 63A-C (1998) 805.

[41] M. Lusher et al., Nucl. Phys. B 413 (1994) 481.

[42] G. Boyd, J. Engels, F. Karsch, E. Laermann, C. Legeland, M. Lutgemeier and B. Petersson, Nucl. Phys. B 469 (1996) 419; hep-lat/9602007.

[43] M. Panero, arXiv:0907.3719 [hep-lat].

[44] V. Gogohia, Gy. Kluge, H. Toki, T. Sakai, Phys. Lett. B 453 (1999) 281.

[45] V. Gogohia, H. Toki, T. Sakai, Gy. Kluge, Inter. Jour. Mod. Phys. A 15 (2000) 45.

[46] G. 't Hooft, Nucl. Phys. B 75 (1974) 461.

[47] A.I. Alekseev, B.A. Arbuzov, Few Body Syst. 40 (2006) 57-70; hep-ph/0407056

[48] Particle Data Group, J. Phys. G: Nucl. Part. Phys. 33 (2006) 1.

[49] V. Gogohia, Gy. Kluge, Phys. Lett. B 477 (2000) 387.

[50] V. Gogohia, M. Prisznyak, Phys. Lett. B 494 (2000) 109.

[51] A.C. Aguilar, D. Binosi, J. Papavassiliou, Phys. Rev. D 78 (2008) 025010; arXiv:0802.1870 [hep-ph].

[52] Ph. Boucaud, J.P. Leroy, A. Le Yaouanc, J. Micheli, O. Péne, J. Rodríguez-Quintero, JHEP 0806 (2008) 099; arXiv:0803.2161 [hep-ph]. 
[53] R. Alkofer, L. von Smekal, Phys. Rep. 353 (2001) 281.

[54] C.S. Fischer, A. Maas, J.M. Pawlowski, arXiv:0801.1987 [hep-ph].

[55] S.P. Sorella, arXiv:0905.1010 [hep-th].

[56] D. Dudal, J.A. Gracey, S.P. Sorella, N. Vandersickel, H. Verschelde, Phys. Rev. D 78 (2008) 065047.

[57] D. Zwanziger, arXiv:0904.2380 [hep-th].

[58] K.-I. Kondo, CHIBA-EP-177, arXiv:0907.3249 [hep-th].

[59] A. Cucchieri, T. Mendes, arXiv:0904.4033 [hep-lat].

[60] A. Cucchieri, T. Mendes, Phys. Rev. Lett. 100 (2008) 241601.

[61] V.G. Bornyakov, V.K. Mitrjushkin, M. Müller-Preussker, arXiv:0812.2761 [hep-lat].

[62] I.L. Bogolubsky, E.-M. Igenfritz, M. Müller-Preussker, A. Sternbeck, Phys. Lett. B 676 (2009) 69.

[63] M.E. Peskin, D.V. Schroeder, An Introduction to Quantum Field Theory, (Addison-Wesley, The Advanced Book Program, 1995)

[64] J.C. Taylor, Nucl. Phys. B 33 (1971) 436.

[65] G. 't Hooft, Nucl. Phys. B 33 (1971) 173.

[66] V.Sh. Gogokhia, Phys. Rev. D 40 (1989) 4157.

[67] V.Sh. Gogokhia, Phys. Rev. D 41 (1990) 3279.

[68] H. Pagels, Phys. Rev. D 15 (1977) 2991.

[69] Statistical Office of the European Communities, http://epp.eurostat.ec.europa.eu

[70] ENERGY FOR THE FUTURE - The Nuclear Option, A position paper of the EPS, www.eps.org

[71] V. Gogokhia, arXiv:hep-ph/0508224.

[72] F.D. Steffen, Eur. Phys. J. C 59 (2009) 557.

[73] M.K. Gaillard, B. Zumino, Eur. Phys. J. C 59 (2009) 213.

[74] S. Weinberg, UTTG-10-96, arXiv:astro-ph/9610044.

[75] C. Quigg, FERMILAB-PUB-09/230-T, arXiv:0905.3187 [hep-ph].

[76] WMAP Collaboration, E. Komatsu et al., Astrophys. J. Suppl. 180 (2009) 330.

[77] F.R. Urban, A.R. Zhitnitsky, arXiv:0909.2684 [astro-ph.CO].

[78] K.A. Milton, arXiv:hep-th/0406024

[79] M.D. Roberts, arXiv:hep-th/0012063.

[80] L.H. Ford, arXiv:0911.3597 [quant-ph].

[81] G.L. Klimchitskaya, V.M. Mostepanenko, arXiv:quant-ph/0609145.

[82] S.-S. Xue, Phys. Lett. B 508 (2001) 211.

[83] S.-S. Xue, Phys. Rev. D 68 (2003) 013004.

[84] M. Scandurra, arXiv:hep-th/0104127.

[85] J.D. Bjorken, S.D. Drell, Relativistic Quantum Mechanics (McGraw-Hill Book Company, 1978).

[86] C. Itzykson, J.-B. Zuber, Quantum Field Theory (McGraw-Hill Book Company, 1984).

[87] J.I. Kapusta, C. Gale, Finite-Temperature Field Theory (Cambridge University Press, 2006). 